\title{
A SWITCHED-ANTENNA NADIR-LOOKING INTERFEROMETRIC SAR ALTIMETER FOR TERRAIN-AIDED NAVIGATION
}

\author{
Inchan Paek ${ }^{1}$, Jonghun Jang ${ }^{2}$, Joohwan Chun ${ }^{3}$ and Jinbae $\mathrm{Suh}^{3}$ \\ ${ }^{1}$ PGM Image Sensor Centre, Hanwha Thales, Kyunggi-do, Korea \\ ic.paek@hanwha.com \\ ${ }^{2}$ Agency for Defense Development, Daejeon, Korea \\ ${ }^{3}$ Department of Electrical Engineering, KAIST, Daejeon, Korea \\ chun@kaist.ac.kr
}

\begin{abstract}
Conventional terrain-aided navigation (TAN) technique uses an altimeter to locate the position of an aerial vehicle. However, a major problem with a radar altimeter is that its beam (or pulse) footprint on the ground could be large, and therefore the nadir altitude cannot be estimated accurately. To overcome this difficulty, one may use the nadir-looking synthetic aperture radar (SAR) technique to reduce the along-track beam width, while the cross-track ambiguity is resolved with the interferometry technique. However, the cross-track resolution is still far from satisfactory, because of the limited aperture size of antennas. Therefore, the usual three-antenna array cannot resolve multiple terrain points in a same range bin, effectively. In this paper, we propose a technique that can increase the cross-track resolution using a large number of antennas, but in a switched fashion, not raising hardware cost.
\end{abstract}

\section{KEYWORDS}

TAN, SAR, Altimeter, Switched Array

\section{INTRODUCTION}

Terrain aided navigation (TAN) technique uses an altimeter to find the position and attitude of an aerial vehicle. However, a major problem with a radar altimeter is that its beam (or pulse) footprint on the ground could be large. For example, in Fig. 1, if the beam width is large, the leading edge of the received echo signal would not be from the nadir point $N$.

To overcome this difficulty, one may use the nadir-looking synthetic aperture radar (SAR) technique to reduce the along-track beam width [1], [2], while the cross-track ambiguity is resolved with the interferometry technique [3], [4], [8]. However, the cross-track resolution is still far from satisfactory, because of the limitation in the number of antennas. Therefore, the usual three-antenna array [7] cannot resolve multiple terrain points in a same range bin, effectively. We may remark that the side-looking SAR [6], which gives high resolution, both along and cross tracks, is usually not acceptable for a low-altitude TAN application. In this paper,

Jan Zizka et al. (Eds) : CCSIT, SIPP, AISC, CMCA, SEAS, CSITEC, DaKM, PDCTA, NeCoM - 2016

pp. 151-157, 2016. (C) CS \& IT-CSCP 2016

DOI : $10.5121 /$ csit.2016.60114 
we propose a technique that can increase the cross-track resolution using a large number of antennas, but in a switched fashion, not raising hardware cost.

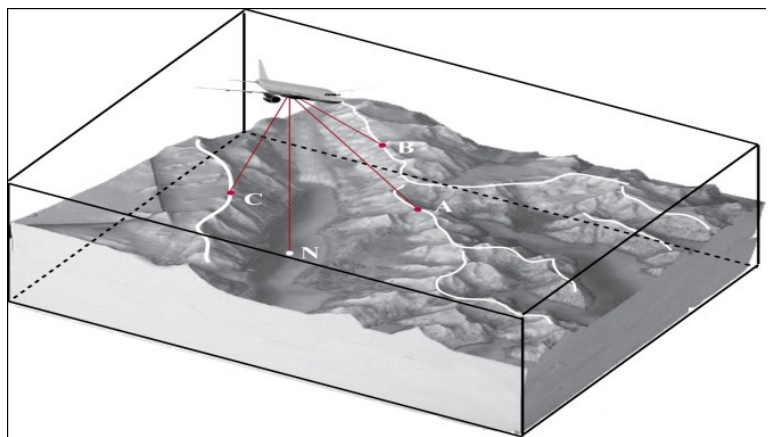

Figure 1: Multipoint-terrain-aided navigation

Figure 2 shows the flight geometry. We assume that there is a separate single transmit antenna, and $N$ receive antennas. Also assume that there are $L$ range bins and $M$ coherent pulses. Then, we can find the range $r$, along-track angle $\theta^{a}$ and the cross-track angle $\theta^{c}$ to a given terrain point, respectively by measuring the echo return time, Doppler and angle of arrival (AOA).

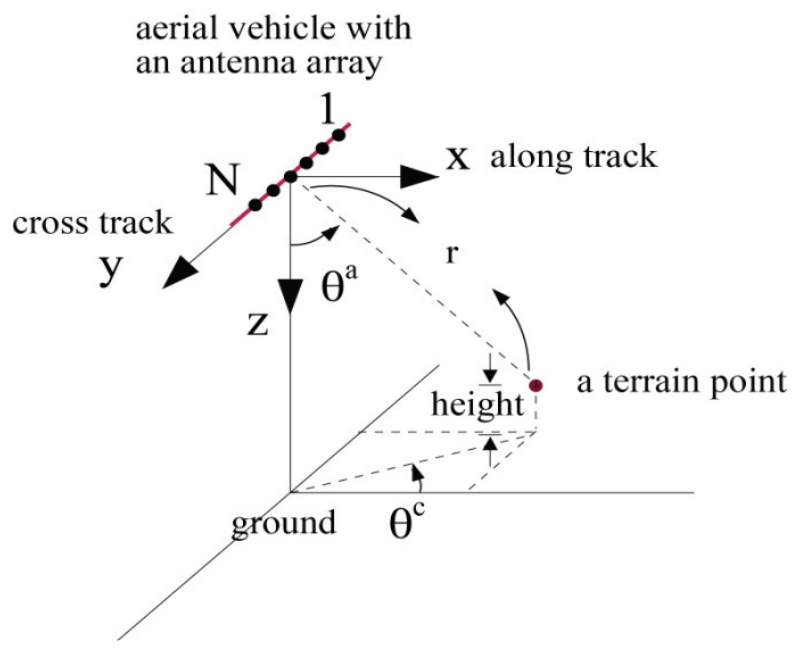

Figure 2: Flight geometry

Fig. 3 shows a 3D data cube, which represents a point target response after the range deramping operation.

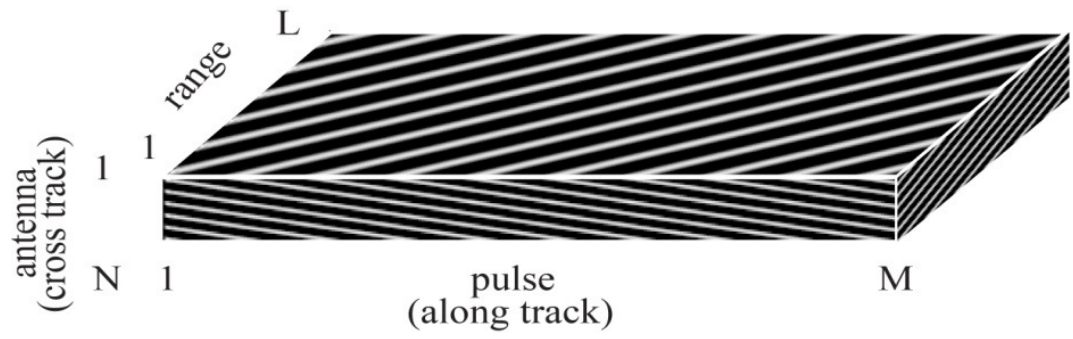

Figure 3: Deramped raw-data for a point target 
The point target position in the 3D can be found by a 3D fast Fourier transform (DFT) of the data cube. The 3D data cube for actual terrain which has an infinite number of point targets will be the superposition of individual point target response, and the reconstruction of the terrain profile in Fig. 1 can be carried out by a 3D FFT of the data cube. From the reconstructed terrain profile, we can estimate the position and attitude of the aerial vehicle.

\section{THE SWITCHED ARRAY ALTIMETER}

To obtain an accurate terrain profile, the number of antennas $N$ must be large, resulting in a large number of RF chains. However, it turns out that a reasonably accurate terrain profile can be found by selecting a small number of $K=N$ antennas, randomly at each pulse. Fig. 4 shows the raw data when only $12 \%$ (Fig 4 b) and 6\% (Fig 4b) of antennas are selected.
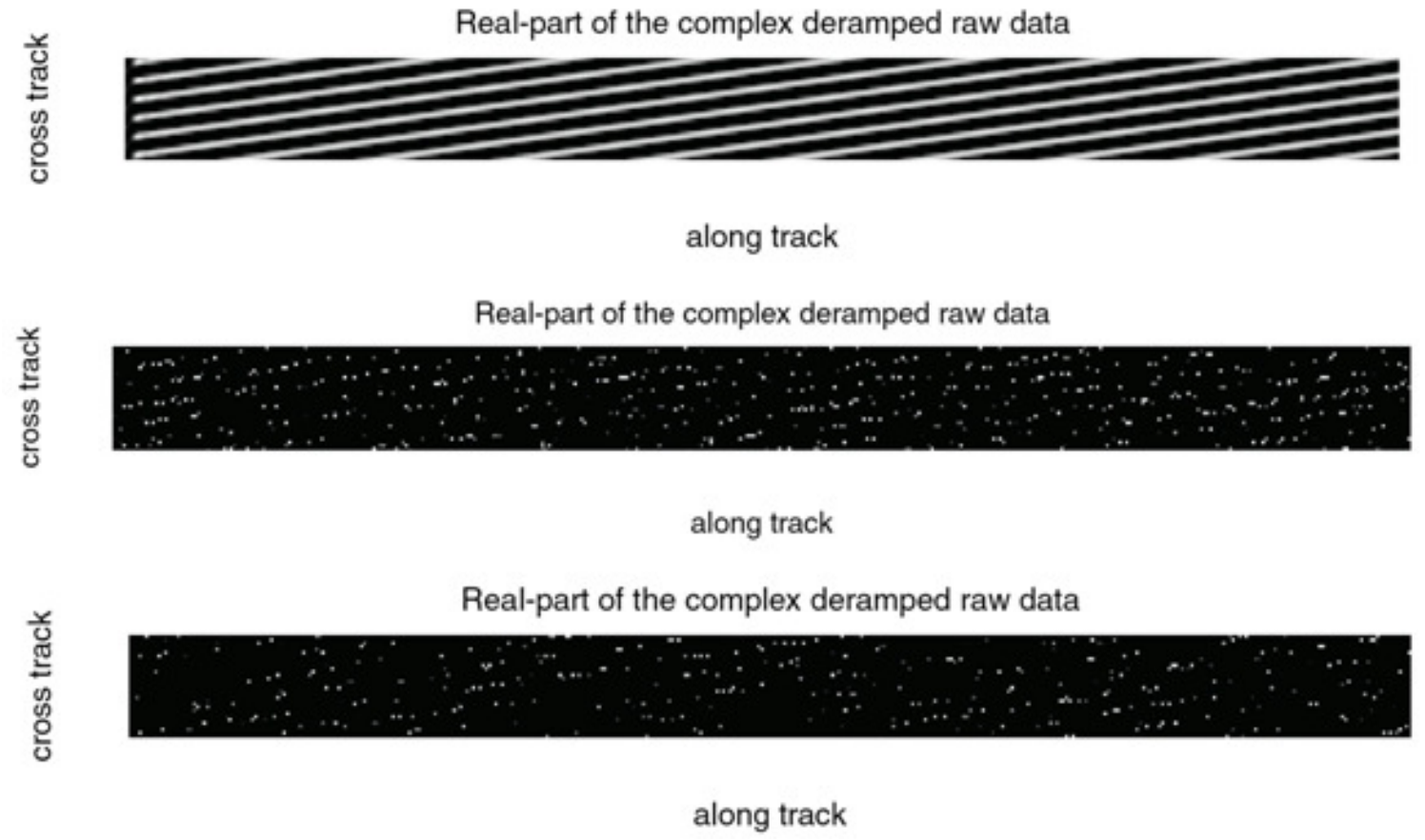

Figure 4. Raw data when (4a) $K=N=32$, (4b) $K=3, N=32$, (4c) $K=2, N=32$

To find the cross-track angle and range to the target, we need to compute the 2D FFTs of the raw data in Fig 4. The results are shown in Fig. 5. Note that the raw data obtained with switching produce reasonably accurate angle and range estimates. 

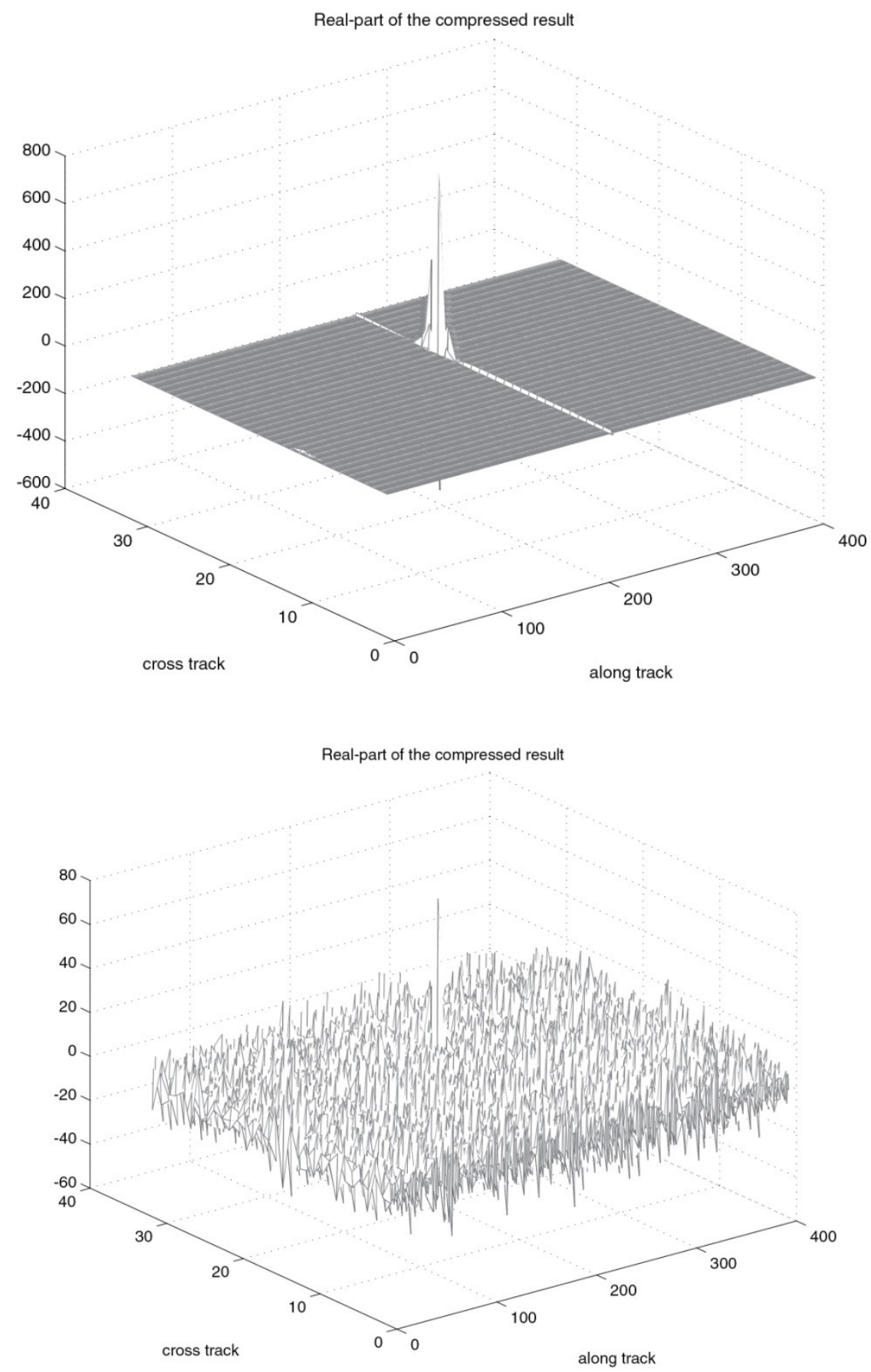


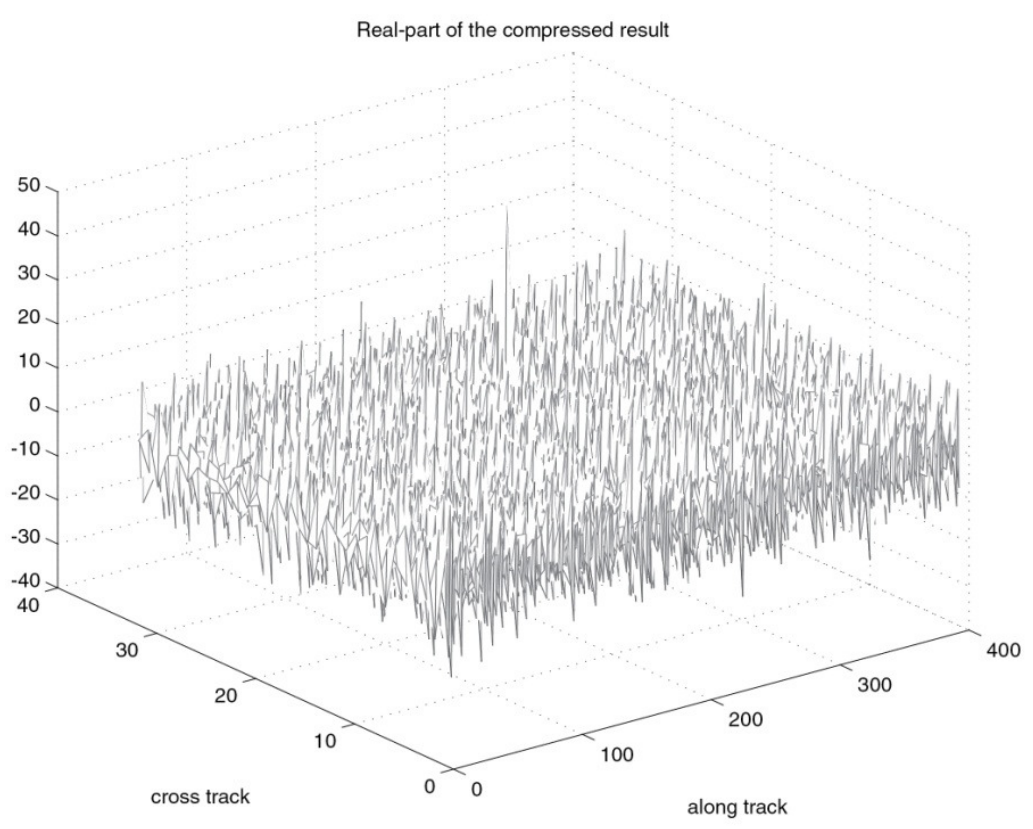

Figure 5. Recovered point target from the raw data obtained by switching. The three plots correspond to the three raw data in Figure 4.

\section{TRACKING ALgORITHM}

We use the usual 15-state Kalman filter [5], [9] to find the attitude and position of the vehicle. In particular, the time-update is carried out using the inertial measurement unit (IMU) data, and the measurement-update is done by comparing the reconstructed terrain profile with the on-board DEM (See Fig. 6). The estimated terrain profile will be particularly accurate at the mountain ridges, which are marked with white curves in Fig 1. Referring to the on-board DEM, we select multiple points (A, B, C in Fig 1) on the ridges, and determine the angles $\theta^{a} \mathrm{~s}$ and $\theta^{c}$ s. Then we determine the ranges $r \mathrm{~s}$ to these points. These measurements are then compared with the predicted measurements $\hat{r}$ to get innovation $e$.

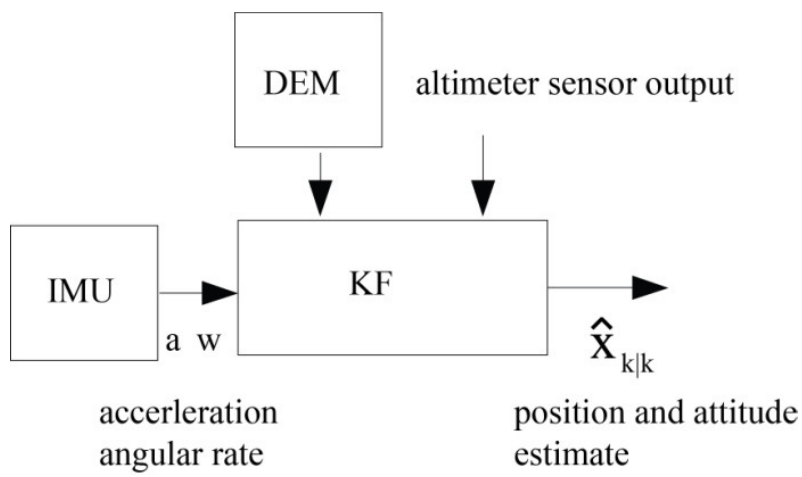

Figure 6. Block diagram of the INS 
Fig 7 shows the detailed computational steps of the proposed inertial navigation system (INS). Our preliminary simulation results show that the convergence of the state estimate is quite faster with the proposed multi-point TAN than with the usual single point TAN.

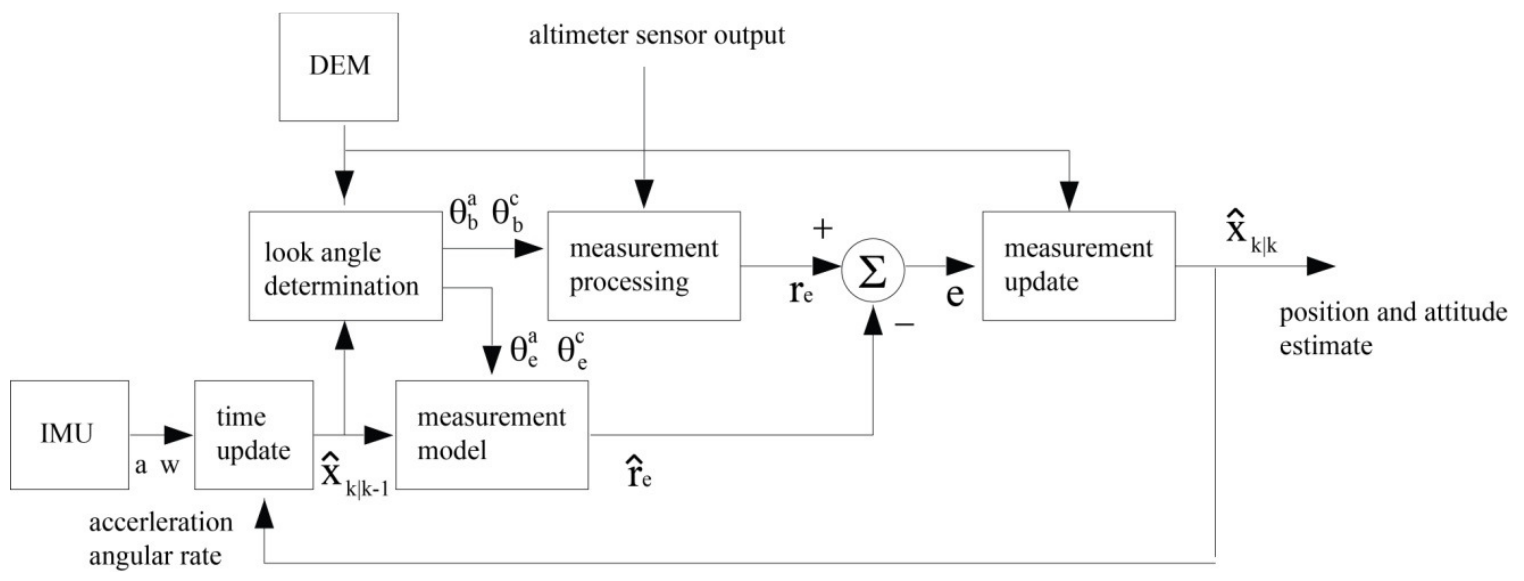

Figure 7. The proposed INS

\section{CONCLUSIONS}

We have proposed a switched antenna interferometric SAR TAN algorithm that can overcome the drawback of conventional interferometric SAR TAN. The proposed technique uses a small number of RF channels which are switched to a large number of antennas. Therefore, our proposed technique will give a high cross - track resolution comparable to the along-track SARbased resolution, not raising hardware cost much.

\section{ACKNOWLEDGEMENTS}

This work was supported in part by Hanwha Thales in 2014-2015.

\section{REFERENCES}

[1] R. Raney, "The Delay/Doppler radar altimeter," IEEE Trans. on Geosci. and Remote Sensing,Vol 36, No 5, Sep. pp. 1578-1588, 1998

[2] R. Raney and J. Jensen, "An airborne CryoSat prototype: The D2P radar altimeter," Proceedings of the International Geoscience and Remote Sensing Symposium, Toronto, pp. 1765-1767, 2002

[3] J. Jensen, "Angle measurement with a phase monopulse radar altimeter," IEEE Trans. On Antennas Propagat., vol 47, No 4, pp. 715-724, 1999.

[4] J. Hager, "Interferometric synthetic aperture radar altimeter," Assignee: Honeywell Inc, US Patent $6,025,800$, Feb 2000

[5] S. Carreno, P. Wilson, P. Ridao, and Y. Petillot, "A survey on terrain based navigation for auvs," MTS/IEEE OCEANS, Seattle, 2010. 
[6] J. Curlander and R. McDonough, Stnthetic Aperture Radar, Systems and Signal Processing, John Wiley, New York, 1991.

[7] H. Jenkins, Small-Aperture Radio Direction Finding, Artech House,Boston, 1991.

[8] J. Chun, S. Choi, I. Paek, D. Park and K. Yoo, "End-to-end design consideration of a radar altimeter for terrain-aided navigation," Proc. SPIE 8891, SAR Image Analysis, Modeling, and Techniques XIII, 889108 Oct. 2013.

[9] D. Titterton and J. Weston, Strapdown inertial navigation technology, second eds, IEE, 2004. 